\title{
Correction: Dynamic classification of personal microenvironments using a suite of wearable, low-cost sensors
}

\author{
Casey Quinn · G. Brooke Anderson · Sheryl Magzamen · Charles S. Henry · John Volckens
}

Published online: 5 March 2020

(C) The Author(s), under exclusive licence to Springer Nature America, Inc. 2020

Correction to: Journal of Exposure Science \& Environmental Epidemiology

https://doi.org/10.1038/s41370-019-0198-2
The original version of this Article featured an incorrect supplementary figure file. This error has been rectified in the PDF and HTML versions of this Article. 